\title{
Perfil dos resíduos industriais enviados às blendeiras fluminenses para tratamento destinado ao coprocessamento
}

Diversos setores da indústria e do comércio brasileiros têm destinado os seus resíduos para serem tratados em unidades de homogeneização e mistura no Estado do Rio de Janeiro conhecidas como blendeiras. O presente estudo tem como objetivo traçar um perfil das principais características dos resíduos industriais que são enviados às blendeiras para tratamento, visando o seu aproveitamento como combustível e matéria-prima na produção de clínquer. A metodologia consistiu, primeiramente, na busca de documentos que constam do processo de licenciamento e controle ambiental no órgão licenciador dessas unidades. Baseado nas informações obtidas nos registros de movimentações de resíduos dos anos de 2010 a 2015, foi realizada a classificação pela origem, de acordo com o Estado da federação, por estado físico, por quantidade, pela NBR ISO 10004, abrangendo a classe e o tipo de periculosidade e, por fim, a sua potencial utilidade no coprocessamento. Também foram registrados: o percentual dos principais produtos da blendagem e os destinos dos resíduos e rejeitos gerados no processo. Como resultado obteve-se o seguinte perfil: os resíduos são originados, majoritariamente, no Estado de São Paulo, pelo setor portuário. Apresentam-se, em sua maioria, no estado sólido, e classificam-se como perigosos e tóxicos e, serão utilizados, principalmente, para geração de energia. Conclui-se que o perfil esboçado contribuiu significativamente para o entendimento da toxicidade desses resíduos e servirá como base para outros estudos que abordem os impactos ambientais e à saúde humana na cadeia do coprocessamento.

Palavras-chave: Resíduos Industriais; Classificação; Toxicidade; Blendagem; Coprocessamento.

\section{Profile of industrial waste sent to the fluminese blenders for treatment intended for co-processing}

\begin{abstract}
Several sectors of Brazilian industry and commerce have destined their residues to be treated in homogenization and mixing units in the State of Rio de Janeiro known as blendeiras. The present study aims to outline a profile of the main characteristics of industrial waste that are sent to blending plants for treatment, aiming at their use as fuel and raw material in the production of clinker. The methodology consisted, first, in the search for documents that are part of the licensing and environmental control process in the licensing agency of these units. Based on the information obtained in the records of waste movements from the years 2010 to 2015 , classification by origin was made, according to the state of the federation, by physical state, by quantity, by NBR ISO 10004 , covering the class and type dangerousness and, finally, its potential usefulness in co-processing. The following were also recorded: the percentage of the main blending products and the destinations of the residues and tailings generated in the process. As a result, the following profile was obtained: the waste originates, mostly, in the State of São Paulo, through the port sector. Most of them are presented in solid state, and are classified as dangerous and toxic and will be used mainly for power generation It is concluded that the outlined profile contributed significantly to the understanding of the toxicity of these residues and will serve as a basis for other studies that address the environmental and human health impacts in the co-processing chain.
\end{abstract}

Keywords: Industrial waste; Classification; Toxicity; Blending; Co-processing.

Topic: Engenharia Ambiental

Reviewed anonymously in the process of blind peer

David Barreto de Aguiar (D)

Universidade do Estado do Rio de Janeiro, Brasil

http://lattes.cnpq.br/7229051875249959

http://orcid.org/0000-0003-1233-0253

david.aguiar@ifri.edu.br

Ubirajara Aluizio de Oliveira Mattos it

Universidade do Estado do Rio de Janeiro, Brasil

http://lattes.cnpq.br/4965089463046103

http://orcid.org/0000-0002-2689-2110

ubirajaraaluizio@yahoo.com.br

Victor Paulo Peçanha Esteves

Universidade Federal do Rio de Janeiro, Brasi

http://lattes.cnpq.br/7858887034547590

http://orcid.org/0000-0002-6812-4558

victoresteves@poli.ufrj.br

d

DOI: 10.6008/CBPC2179-6858.2021.007.0022
Received: 06/07/2021

Approved: 28/07/2021
Referencing this:

AGUIAR, D. B.; MATTOS, U. A. O.; ESTEVES, V. P. P... Perfil dos resíduos industriais enviados às blendeiras fluminenses para tratamento destinado ao coprocessamento. Revista Ibero Americana de Ciências Ambientais, v.12, n.7, p.236-243, 2021. DOI:

http://doi.org/10.6008/CBPC2179-6858.2021.007.0022 


\section{INTRODUÇÃO}

A técnica do coprocessamento tem sido empregada mundialmente com a finalidade de reduzir o consumo de combustível fóssil e matérias-primas na fabricação de clínquer. Trata-se da utilização de rejeitos de atividades industriais, pneus e até mesmo resíduos sólidos urbanos como combustíveis alternativos e matérias-primas nesse processo produtivo (LAMAS et al., 2013).

A literatura científica mundial tem registrado os seguintes resíduos como materiais substitutivos das matérias-primas extraídas diretamente de jazidas: diversos tipos escórias de alto forno, cinzas volantes, argilas, lodos carbonizados, areias de fundição, resíduos que são fontes de ferro, de cálcio, de silício e alumínio (ÇANKAYA et al., 2019; CHEN et al., 2015; GARCÍA-GUSANO et al., 2015; GIORGIOPOULOU et al., 2018; HOSSAIN et al., 2017; SONG et al., 2016).

Como combustíveis alternativos mais utilizados no coprocessamento, a literatura científica registra os seguintes resíduos: combustível derivado de pneu, lodo biológico e seco, combustível derivado do resíduo, resíduo industrial preparado, óleos usados, tintas, solventes, combustíveis líquidos, plásticos papel, papelão e resíduos agrícolas (ÇANKAYA et al., 2019; GIORGIOPOULOU et al., 2018; HOSSAIN et al., 2017; SONG et al., 2016; ZHANG et al., 2016).

Cabe ressaltar que em países como o Japão os resíduos, antes de serem coprocessados, passam por um rigoroso processo de classificação (LI et al., 2014) e que, na maioria dos casos europeus, os riscos no preparo dos resíduos para o coprocessamento são minimizados através da automação dos processos (MILANEZ et al., 2009).

No Brasil, a NBR ISO 10004 (ABNT, 2004) é um instrumento normativo que trata da classificação dos resíduos. Segundo a norma, os mesmos podem ser classificados como Perigosos (Classe I) e Não perigosos (Classe II).

Os resíduos perigosos são aqueles que apresentam pelo menos uma dessas características: inflamabilidade, corrosividade, reatividade, toxicidade e patogenicidade. Os não perigosos ainda são subclassificados como: não inertes (classe IIA), que possuem propriedades como biodegradabilidade, combustibilidade ou solubilidade em água. Já os inertes (classe IIB) não alteram as suas características em contato com a água (ABNT, 2004).

Setores da indústria e do comércio brasileiros como os de papel e celulose, alumínio, automotores, química, petroquímica, energia elétrica, siderurgia, pneus, metalurgia, metalurgia e embalagens têm utilizado o coprocessamento como forma de destruição dos seus resíduos (ABCP, 2020a).

Em 2018, a geração de resíduos industriais no Brasil foi de 1.276.199.686 toneladas de sólidos, $490.650 \mathrm{~m}^{3}$ dos resíduos líquidos e 3.141 .512 unidades, tendo a indústria química como principal setor responsável por essa geração.

Para que esses resíduos possam ser aproveitados nesta atividade, como fontes de energia ou matérias-primas alternativas na indústria de cimento, os mesmos precisam ser preparados em unidades industriais de mistura e homogeneização conhecidas como blendeiras (MILANEZ et al., 2009). 
Estima-se que haja no Brasil, aproximadamente, vinte plantas de blendagem em operação (AGUIAR et al., 2021), no município de Magé, situado na Região Metropolitana do Estado do Rio, situam-se duas importantes blendeiras que preparam os resíduos para serem coprocessados em fornos de clínquer no polo cimenteiro de Cantagalo, um dos mais importantes do país, que fica localizado na Região Serrana do mesmo estado.

Conforme descreveu Milan (2017), muitos líquidos, óleos e produtos químicos como tintas e solventes, além de óleos refrigerantes e de motor, são tóxicos. Milanez et al. (2009) discutiram riscos sanitários como a exposição a vapores e odores das diversas etapas de mistura e homogeneização de resíduos perigosos, nesta atividade (ROCHA et al., 2011).

Pinto Júnior et al. (2009) analisaram as condições de trabalho e saúde a partir dos relatos dos trabalhadores envolvidos na atividade de coprocessamento de resíduos tóxicos em fornos de cimenteiras de Cantagalo e constataram falhas no processo de trabalho, recaindo sobre os trabalhadores o ônus do adoecimento. Estes resíduos chegam às blendeiras de diversas formas: a granel, em caminhões basculantes, ou acondicionados em tambores, bombonas, contêineres, big bags e embalagens diversas (INEA, 2020).

O processo de blendagem é responsável por produzir blends de resíduos com poder calorífero na faixa de 1800 a $3500 \mathrm{Kcal} / \mathrm{kg}$, (blends sólidos) e de 2000 a $10500 \mathrm{Kcal} / \mathrm{kg}$, (blends líquidos), com vistas a serem coprocessados (INEA, 2020). Rocha et al. (2011) mencionam que o coprocessamento é um campo amplo de investigação e indicam que cada tipo de resíduo coprocessado deve ser objeto de estudo, devido às suas características físicas e químicas.

No entanto, a literatura científica brasileira carece de estudos que se aprofundem no conhecimento detalhado dos resíduos que são tratados nas blendeiras, para que possam ser compreendidos os potenciais riscos e impactos que podem ser causados ao meio ambiente e à saúde humana, com vistas ao aperfeiçoamento dos processos de controle ambiental e fiscalização.

Em face dos argumentos expostos, o presente estudo tem por objetivo traçar um perfil das principais características dos resíduos industriais que são enviados às blendeiras para tratamento, visando o seu aproveitamento como combustível e matéria-prima na produção de clínquer.

\section{METODOLOGIA}

Os dados do trabalho foram obtidos a partir da análise de dois processos de licenciamento ambiental no órgão estadual de meio ambiente de duas plantas de blendagem, situadas no município de Magé, Região Metropolitana do Estado. Neste processo, foram encontrados dados dos anos de 2010 a 2015.

Foram analisadas 181 autorizações ambientais de movimentação de resíduos, três pareceres técnicos e um plano de trabalho, que continham informações sobre os diversos tipos de resíduos industriais utilizados na preparação dos compostos energéticos conhecidos como blends. Cabe ressaltar que a busca e recuperação de informação contemplaram documentos que se classificam na categoria "Literatura Cinzenta" (BOTELHO et al., 2015):

Diz respeito a publicações não convencionais e não comerciais, semipublicadas, difíceis de 
encontrar em canais tradicionais de distribuição [...]. Apresentam informação e conhecimento altamente atualizados e mais detalhados, alcançam um público reduzido e não são determinadas apenas por interesses comerciais.

Com os dados encontrados nas duas plantas, foi construído um perfil médio anual da quantidade de resíduos tratados em toneladas. Os resíduos foram identificados por tipo e, posteriormente, separados em dois grandes grupos por estado físico: os sólidos/semissólidos e os líquidos, com a finalidade de rastrear os resíduos que seriam transformados em blends sólidos e líquidos.

Buscou-se também identificar os resíduos gerados por setores da economia, estimando o percentual de contribuição desses setores. Registrou-se, também, a origem territorial dos resíduos por Estado da federação. A seguir, obedecendo à separação dos resíduos pelo estado físico em dois grupos, buscou classificar os tipos de resíduos de acordo com a NBR ISO 10004 em: Perigosos (Classe I), Não Perigosos não inertes (Classe IIA) e Não perigosos inertes (Classe IIB).

O tipo de periculosidade encontrado em cada resíduo perigoso foi classificado conforme a referida norma, a saber: tóxico, altamente tóxico, inflamável, corrosivo e reativo. Foi admitido que um mesmo tipo de resíduos tenha dois ou mais formas de periculosidade. A classificação que obteve percentual menor que $1 \%$ não foi considerada.

Foi classificado, também, o potencial do resíduo quanto a sua utilização futura na produção de clínquer, se como combustível ou matéria-prima, conforme a classificação da Associação Brasileira de Cimento Portland (2020b). Os resultados da classificação pela norma, pelo tipo de periculosidade e pela potencial utilização do resíduo no coprocessamento foram ilustrados por gráficos do Excel.

Por fim, foi estimado o percentual de resíduos e rejeitos da blendagem que seguiram para outras formas de tratamento e destinação, como: reciclagem, estações de tratamento de efluentes, incineração e aterro sanitário.

Este trabalho se limita apenas aos resíduos industriais e não aborda o caso dos pneus inservíveis, que, apesar de utilizados no coprocessamento em fornos de cimento, não são armazenados e tratados nas blendeiras estudadas. O trabalho tampouco leva em consideração o detalhamento de cada etapa do processo de blendagem outrora abordado por Aguiar et al. (2021).

\section{RESULTADOS E DISCUSSÃO}

O levantamento realizado constatou que o recebimento médio de resíduos industriais foi de 79.015,82 toneladas por ano, distribuídos em 174 tipos de resíduos para serem tratados, destes, 89\% da massa apresentam-se no estado sólido e/ou semissólido, e 11\% no estado líquido.

Os percentuais de geração, por regiões do país, foram os seguintes: Sudeste (95\%), Nordeste (4\%) e Centro-Oeste (1\%). Na Região Sudeste o Estado de São Paulo foi responsável por 90\% dos resíduos gerados.

Os resíduos industriais foram gerados por 61 atividades econômicas dos setores da indústria e comércio brasileiros com os seguintes percentuais: setor portuário $(45,94 \%)$, óleo e gás $(20,48 \%)$, gerenciamento de resíduos e reciclagem $(11,13 \%)$, energia elétrica $(5,82 \%)$, indústria química em geral $(3,47 \%)$, lubrificantes $(3,11 \%)$, tintas e vernizes $(2,80 \%)$, automotivo $(2,11 \%)$, aviação $(1,31 \%)$ e outras 
atividades menores que $1 \%(3,84 \%)$.

Os diversos tipos de resíduos sólidos industriais utilizados na produção dos blends sólidos são os seguintes: solo contaminado $(39,83 \%)$, borra oleosa $(16,35 \%)$, graxas $(8,89 \%)$, resíduos químicos e reagentes diversos $(6,59 \%)$, areia contaminada $(4,08 \%)$, borra de tintas - base de água $(2,96 \%)$, base solvente $(2,29 \%)$ -, lodo de ETE (2\%), resinas (1,94\%), brita contaminada (1,87\%), demais resíduos menores que $1 \%(11,59 \%)$.

Já os resíduos líquidos apresentaram a seguinte distribuição percentual: lubrificantes (39,43\%), solventes (23,54\%), etanol (9,98\%), sulfonato de alquibenzeno $(5,33 \%)$, álcoois etoxilados $(5,25 \%)$, sulfato de ferro $(4,25 \%)$, diesel $(2,69 \%)$, ácido fosfórico $(2,04 \%)$, formaldeído $(1,61 \%)$, ácido graxo $(1,32 \%)$, óleos $(1,32 \%)$, água oleosa $(1,01 \%)$, demais resíduos líquidos menores que $1 \%(5,23 \%)$ (INEA, 2020). Passando-se à classificação dos resíduos pela NBR ISO 10004, constatou-se que em ambos os grupos, sólidos e líquidos, a maioria dos resíduos que chega à blendagem é classificada como perigosa (Classe I).

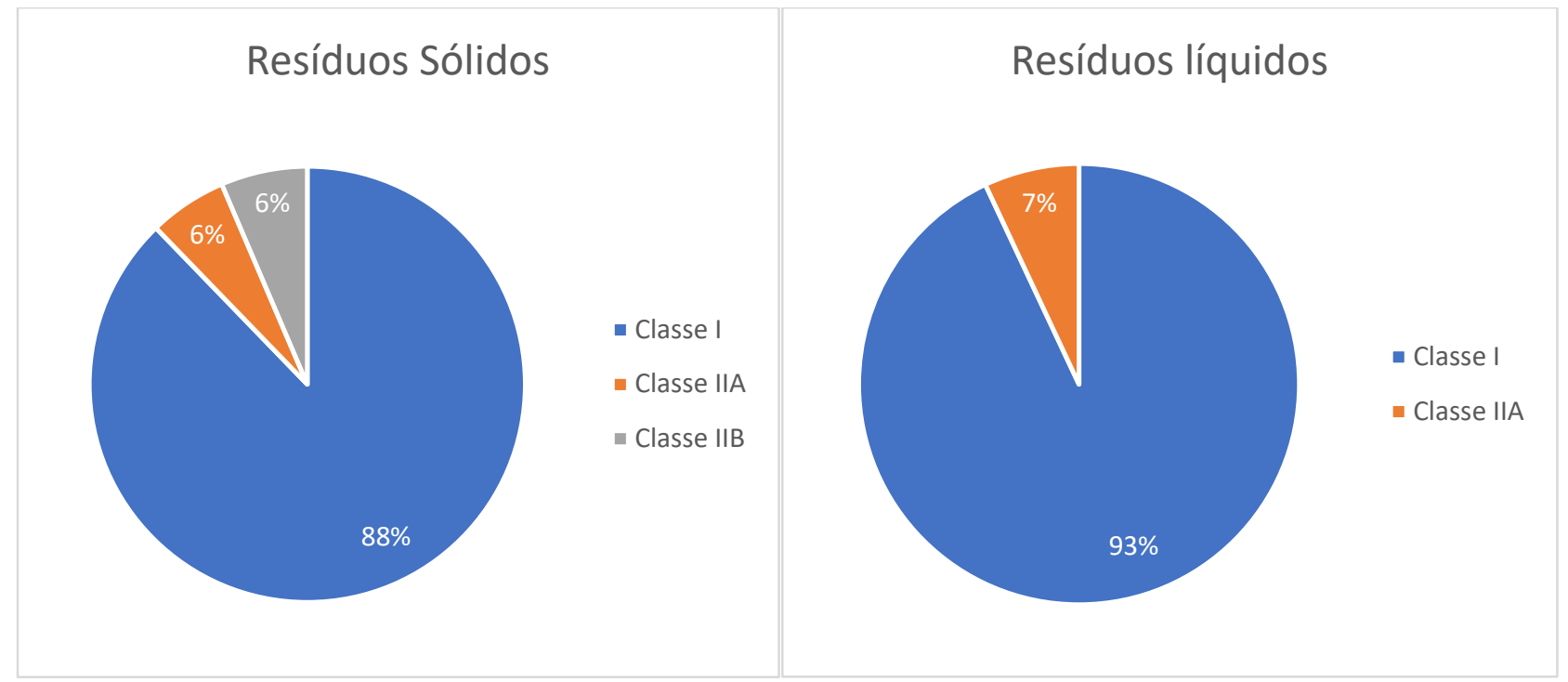

Figura 1: Classificação dos resíduos industriais quanto à classificação pela NBR ISO 10004.

Baseando-se na NBR ISO 10004 (ABNT, 2004), o tipo de periculosidade predominante encontrado nos resíduos classe I é a toxicidade (Figura 2). Logo, a homogeneização, que visa aumentar o poder calorífero dos blends sólidos, é mais trabalhada nas etapas de blendagem, pois envolve mistura um tratamento físico, utilizando técnicas de trituração e picagem, bem como processos físico-químicos envolvendo a mistura da massa de resíduos com serragem e cal, visando a reduzir a umidade, tornando o blend sólido mais fluido para a queima (INEA, 2020).

Notou-se que nos resíduos líquidos a característica de inflamabilidade aumenta em relação aos sólidos, apesar da toxicidade continuar a ser a característica predominante.

Quanto à análise da possível funcionalidade dos resíduos industriais para o coprocessamento, os sólidos apresentaram maior equilíbrio quanto a servirem como combustível ou matéria-prima, com pequeno predomínio percentual para a primeira função como destaca a Figura 3. Já os resíduos industriais líquidos têm função potencial predominante para a geração de energia.

Quanto aos produtos da blendagem, o estudo registrou uma produção média anual total de 30.650 toneladas, que é percentualmente dividida pelos seguintes tipos de blends, conforme a tabela 1. 


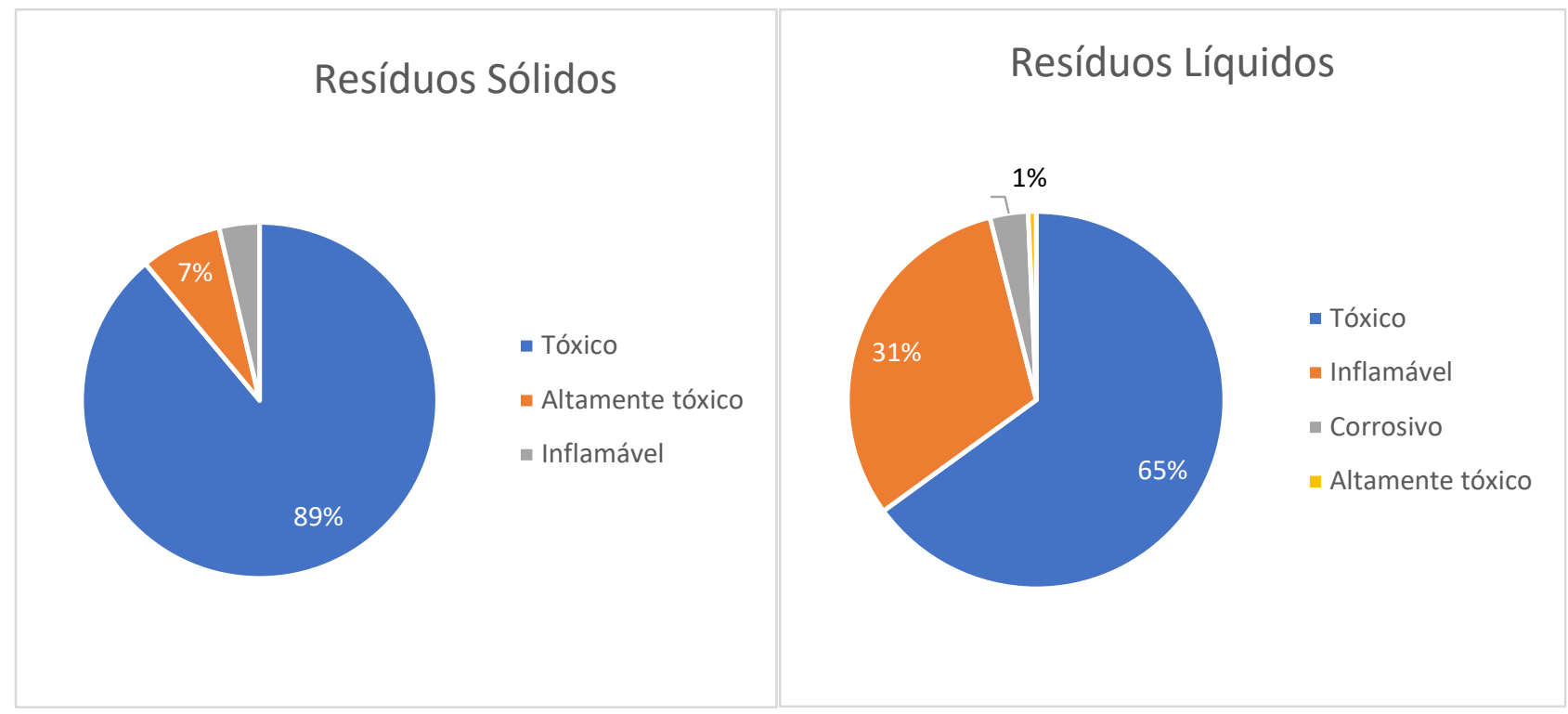

Figura 2: Predominância da toxicidade dos resíduos quanto à periculosidade.

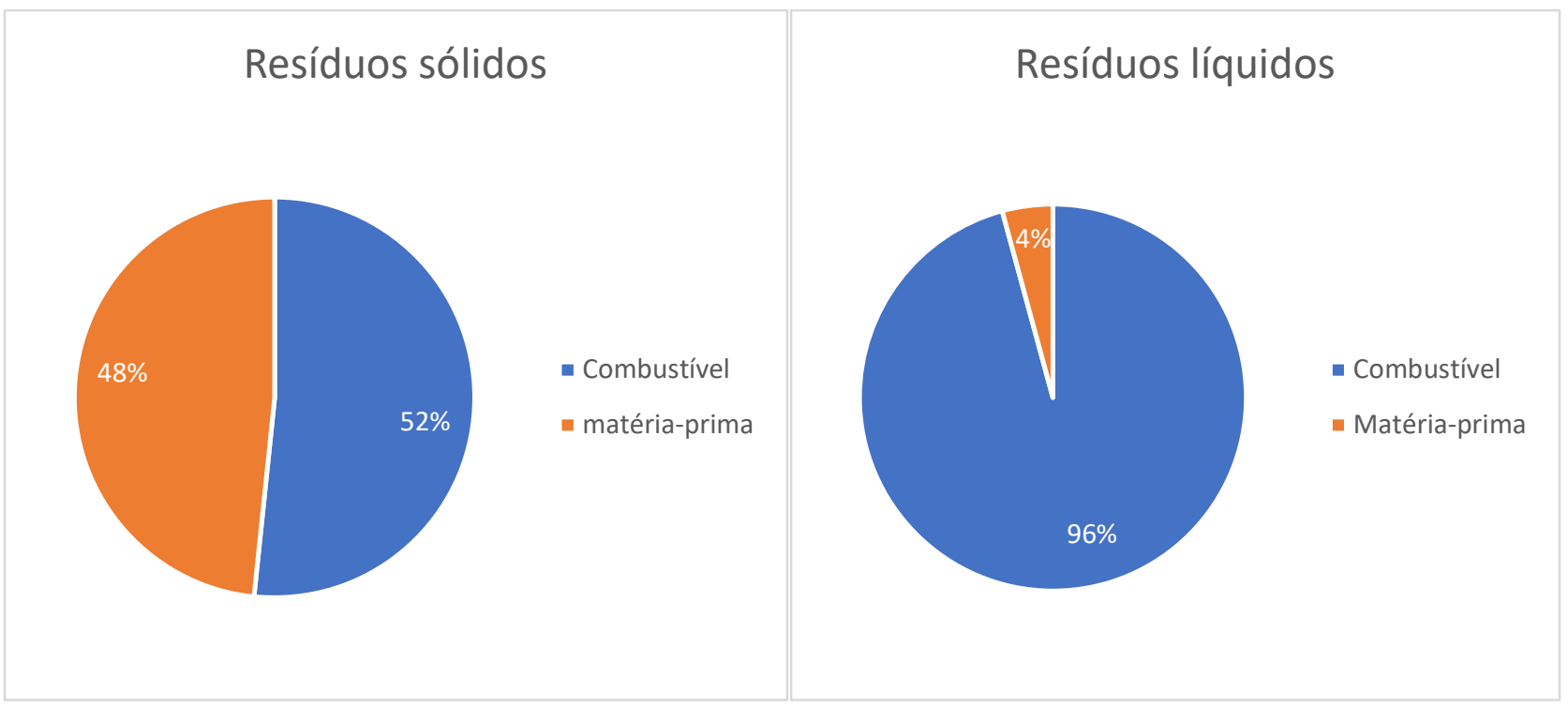

Figura 3: Potencial funcionalidade dos resíduos nos fornos de clínquer.

Tabela 1: Percentuais dos produtos produzidos na blendagem.

\begin{tabular}{ll}
\hline Tipos de blend produzido & $(\%)$ \\
\hline Blend sólido energético & 65 \\
Blend matéria-prima & 22 \\
Blend líquido & 13 \\
\hline
\end{tabular}

Fonte: INEA (2020).

Corroborando com os resultados encontrados nestes estudos, a toxicidade humana foi o principal impacto encontrado por Aguiar et al. (2021) quando aplicaram a Avaliação do Ciclo de Vida na cadeia do coprocessamento na produção de uma tonelada de clínquer, que considerou a entrada desses blends de resíduos produzidos nessas mesmas blendeiras como substituto parcial dos resíduos tradicionais nos fornos de clínquer.

Por fim, considerando que a entrada anual de resíduos foi em média 79.015,82 toneladas e a produção anual de blends é de 30.650 toneladas, constatou-se que sobraram 48.365,82 toneladas de resíduos e rejeitos, ou seja, o equivalente a $62 \%$, que são enviados para outros destinos, conforme os 
percentuais demonstrados na tabela 2 .

Tabela 2: Percentuais de resíduos e rejeitos da blendagem e suas destinações finais.

\begin{tabular}{lll}
\hline Tipos de resíduos ou rejeitos & Destino & (\%) \\
\hline Sobras de processo e triagem não coprocessáveis & Aterro sanitário & 51 \\
Tambores e sucatas metálicas & Reciclagem em siderurgias & 30,8 \\
Paletes e madeira & Reciclagem externa/olarias & 18 \\
Lâmpadas fluorescentes & Recuperação e reciclagem & 0,1 \\
Resíduos de laboratório & Incineração externa & 0,1 \\
\hline
\end{tabular}

Fonte: INEA (2020).

\section{CONCLUSÕES}

Tendo em vistas as informações levantadas na etapa de blendagem, incluindo a sua origem, tipologias, classificações, potenciais funcionalidades nos fornos de clínquer, bem como dos produtos, resíduos e rejeitos gerados no processo, conclui-se que o perfil esboçado contribuiu significativamente para o entendimento da toxicidade desses resíduos e servirá como base para futuros estudos que analisem a relação entre os impactos ambientais e à saúde humana na cadeia do coprocessamento com este tipo de periculosidade incorporada aos blends.

Contudo, o coprocessamento não se mostrou uma técnica de solução definitiva para eliminação dos resíduos industriais do ponto de vista ambiental, pois, a blendagem, que é o processo de preparação dos resíduos pré-queima, não aproveitou a maioria dos resíduos recebidos em blends, confirmando uma fragilidade neste elo da cadeia de coprocessamento, como se mostrou também uma grande geradora de resíduos e rejeitos que precisaram ser remetidas a outros destinos.

\section{REFERÊNCIAS}

AGUIAR, D. B.; MATTOS, U. A. O.; ESTEVES, V. P. P.. Avaliação do ciclo de vida na cadeia de coprocessamento. Revista Ibero-Americana de Ciências Ambientais, v.12, n.6, p.377386, 2021. DOI: http://doi.org/10.6008/CBPC2179$\underline{6858.2021 .006 .0031 .12}$

ABCP. Associação Brasileira de Cimento Portland. Coprocessamento: uma solução definitiva para o resíduo. $O$ que é? São Paulo, 2020a.

ABCP. Associação Brasileira de Cimento Portland. Coprocessamento: unidades de coprocessamento no Brasil. São Paulo, 2020b.

ABNT. Associação Brasileira de Normas Técnicas. ABNT NBR 10004: resíduos sólidos - classificação. 2 ed. Rio de Janeiro: ABNT, 2004.

BOTELHO, R. G.; OLIVEIRA, C. C.. Literaturas branca e cinzenta: uma revisão conceitual. Ciência da Informação, Brasília, v.44, n.3, p.501-513, 2015.

ÇANKAYA, S.; PEKEY, B.. A comparative life cycle assessment for sustainable cement production in Turkey, Journal of Environmental Management, v.249, 109362, 2019. DOI: https://doi.org/10.1016/i.jenvman.2019.109362

GARCÍA-GUSANO, D.; HERRERA, I.; GARRAÍN, D.; LECHÓN, Y.; CABAL, H.. Life cycle assessment of the Spanish cement industry: implementation of environmental-friendly solutions. Clean Technologies Environmental Policy, v.17, p.59-73, 2015. DOI: https://doi.org/10.1007/s10098-014$\underline{0757-0}$

GIORGIOPOULOU, M.; LYBERATOS, G.. Life cycle assessment of the use of alternative fuels in cement kilns: a case study. Journal of Environmental Management, v.216, p.224-234, 2018. DOI: https://doi.org/10.1016/i.jenvman.2017.07.017

HOSSAIN, M. U.; POON, C. S.; LO, I. M. C.; CHENG, J. C. P.. Comparative LCA on using waste materials in the cement industry: A Hong Kong case study. Resources, Conservation and Recycling, v.120, p.199-208, 2017. DOI: https://doi.org/10.1016/j.resconrec.2016.12.012

INEA. Instituto Estadual do Ambiente. Portal do Licenciamento. Rio de Janeiro: Instituto Estadual do Ambiente, 2020

LAMAS, W. Q.; PALAU, J. C. F.; CAMARGO, J. R.. Waste materials co-processing in cement industry: ecological efficiency of waste reuse. Renewable and Sustainable Energy Reviews, v.19, p.200-207, 2013. DOI: https://doi.org/10.1016/j.rser.2012.11.015

MILAN, M.. Reciclagem de materiais: empresa e conceito. São Paulo: SENAI-SP, 2017. 
MILANEZ, B.; FERNANDES, L. O.; PORTO, M. F. S.. A

coincineração de resíduos em fornos de cimento: riscos para a saúde e o meio ambiente. Ciência \& Saúde Coletiva, v.14, n.6, p.2143-2152, 2009. DOI:

https://doi.org/10.1590/S1413-81232009000600021

PINTO JÚNIOR, A. G. P.; BRAGA, A. M. C. B.. Trabalho e saúde: a atividade da queima de resíduos tóxicos em fornos de cimenteiras de Cantagalo, Rio de Janeiro. Ciência \& Saúde Coletiva, v.14, n.6, p.2005-2014, 2009. DOI: http://dx.doi.org/10.1590/S1413-81232009000600008

ROCHA, S. D. F.; LINS, V. F. C.; SANTO, B. C. E.. Aspectos do coprocessamento de resíduos em fornos de clínquer.
(Revisão da Literatura). Engenharia Sanitária e Ambiental, v.16, n.1, p.1-10, 2011. DOI:

http://dx.doi.org/10.1590/S1413-41522011000100003

SONG, D.; YANG, J.; CHEN, B.; HAYAT, T.; ALSAEDI, A.. Lifecycle environmental impact analysis of a typical cement production chain. Applied Energy, v.164, p.916-923, 2016. DOI: https://doi.org/10.1016/j.apenergy.2015.09.003

ZHANG, L.; MABEE, W, E.. Comparative study on the lifecycle greenhouse gas emissions of the utilization of potential low carbon fuels for the cement industry. Journal of Cleaner Production, v.122, p.102-112, 2016. DOI:

https://doi.org/10.1016/j.jclepro.2016.02.019

A CBPC - Companhia Brasileira de Produção Científica (CNPJ: 11.221.422/0001-03) detém os direitos materiais desta publicação. Os direitos referem-se à publicação do trabalho em qualquer parte do mundo, incluindo os direitos às renovações, expansões e disseminações da contribuição, bem como outros direitos subsidiários. Todos os trabalhos publicados eletronicamente poderão posteriormente ser publicados em coletâneas impressas sob coordenação da Sustenere Publishing, da Companhia Brasileira de Produção Científica e seus parceiros autorizados. Os (as) autores (as) preservam os direitos autorais, mas não têm permissão para a publicação da contribuição em outro meio, impresso ou digital, em português ou em tradução. 